\title{
Spin excitation spectra of integral and fractional quantum Hall systems
}

\author{
Arkadiusz Wójs \\ Department of Physics, University of Tennessee, Knoxville, Tennessee 37996, \\ and Institute of Physics, Wroclaw University of Technology, Wroclaw 50-370, Poland \\ John J. Quinn \\ Department of Physics, University of Tennessee, Knoxville, Tennessee 37996
}

\begin{abstract}
Results are presented of detailed numerical calculations for the spin excitation spectra of a twodimensional electron gas confined in a quantum well of finite width $w$, at magnetic fields corresponding to the fractional and integral fillings of the lowest and of excited Landau levels. Spin waves and skyrmions are identified, and their mutual interactions are studied at filling factors $\nu=\frac{1}{3}, 1,3$, and 5. The smallest skyrmions at $\nu=\frac{1}{3}$ are equivalent to composite fermion charged excitons $X_{\mathrm{CF}}^{ \pm}$. Bose condensates of nearly noninteracting spin waves and Laughlin correlations between finite-size skyrmions are found at $\nu=1$ and $\frac{1}{3}$ and, for $w$ larger than about two magnetic lengths, also at $\nu=3$ and 5. A general criterion for the occurrence of skyrmions at odd integral fillings and at Laughlin fractional fillings of the lowest Landau level is given in terms of the interaction pseudopotentials. This explains the dependence of skyrmion states on $w$ observed in excited Landau levels.
\end{abstract}

PACS numbers: 73.43.-f, 71.10.Pm, 73.21.-b

\section{INTRODUCTION}

The low-energy dynamics of a two-dimensional electron gas (2DEG) in a strong magnetic field $B$ is determined by the particular form of the electron-electron $(e-e)$ interaction in a macroscopically degenerate, partially filled Landau level (LL). 1 In the lowest LL, the short range of this (repulsive) interaction results in a particular form of (Laughlin) correlations. 1 Namely, at each given density $\varrho$, yielding a fractional LL filling $\nu=\varrho \phi_{0} / B=2 \pi \varrho \lambda^{2} \quad\left(\phi_{0}=h c / e\right.$ is a magnetic flux quantum and $\lambda=\sqrt{\hbar c / \rho_{f} \beta}$ is the magnetic length), the electrons tend to avoid5.6 as much as possible (within their Hilbert space severely limited by the LL quantization) the pair eigenstates with the highest repulsion, that is with the smallest relative pair angular momentum $\mathcal{R}=0,1,2, \ldots$ This microscopic property is responsible for variety of macroscopic, experimentally observable effects. A well-know example is the fractional quantum Hall effect|⿴囗十 in which a finite gap for charge excitations at $\nu=\frac{1}{3}, \frac{2}{5}$, etc., causes quantization of the Hall conductance at the universal values, $\sigma_{x y}=\nu e^{2} / h$. Another example is a nonlinear dependence of the spin polarization $\zeta$ on the magnetic field $B$ (or density) near $\nu=1$ (in the systems in which the Zeeman energy $E_{\mathrm{Z}}$ an be made sufficiently small to allow spin-excitations), 14 connected with the existence of particle-like excitations carrying massive spin, generally called skyrmions. 15.17

It is quite remarkable that both features that determine the properties of the 2DEG in the quantum Hall regime - the degenerate LL structure of the singleparticle spectrum and the repulsive interaction between the particles (electrons or holes) in a partially filled LL - repeat at different filling factors $\nu$. Clearly, the situation near all odd integral $\nu=1,3, \ldots$ is very similar if only the cyclotron energy $\hbar \omega_{c}$ is sufficiently large to prevent the inter-LL scattering (and cause decoupling of a partially filled excited LL from the rigid core of completely filled, lower LL's). Less obviously, the Laughlin correlations in a partially filled lowest LL allow (at low energy) the mapping of the original electron system near $\nu=(2 p+1)^{-1}$ onto the system of weakly interacting quasiparticles (QP's) with $(2 p+1)$ times smaller degeneracy $g^{*}$ of their quasi-LL's.18 It is a matter of preference whether the reduced quasi-LL degeneracy $g^{*}=g /(2 p+1)$ is attributed to the fractional charge $\pm e /(2 p+1)$ of theQP's 418 or, as in the composite fermion (CF) picture, 1922 to the partial cancellation of the magnetic field $B$ by the Chern-Simons gauge field. In any case, the effective QP filling factor is $\nu^{*} \approx 1$ (which allows interpretation of the fractional quantum Hall effect of electrons as an integral quantum Hall effect 23 of QP's), with small residual interaction between the (charged) QP's.

The answer to the question if the low-energy spin excitations of all these similar systems are indeed similar (and, e.g., include stable skyrmions) lies in the details of this residual interaction within the relevant (lowest or excited, electron or CF) LL. This question is not at all trivial, as it is known that the repulsive character of this interaction is, by itself, not sufficient to cause similar correlations. For example, unlike in the lowest LL, electrons at the $\nu=\frac{1}{3}$ filling of an excited LL do not form a Laughlin ground state.24 26 Similarly, of the two types of Laughlin QP's, the quasiholes (QH's) do, but the quasielectrons (QE's) do not form a Laughlin ground state at a QP filling of $\nu_{\mathrm{QP}}=\frac{1}{3}$.27 To make things more complicated, the effective 2D interaction in realistic quasi-2D systems depends also on the details of the confinement (in the $z$-direction, perpendicular to the $2 \mathrm{D}$ layer) 28

The spin excitations at $\nu=1$ have been inyestigated both experimentally 140 and theoretically, 2940 and both 
in an extended 2DEG and in finite-size quantum Hall droplets.41 43 Importantly, an exact mapping between the unpolarized electron $(\uparrow-\downarrow)$ and polarized electronvalence-hole $(e-h)$ systems in the lowest LI44 makes $\nu=1$ skyrmion equivalent to a charged multi-exciton 45,46 $\left(X_{K}^{ \pm}\right)$consisting of $K$ neutral excitons bound to an electron or to a hole. This allows cross-interpretation of the results obtained from the (experimental and theoretical) studies of spin and optical excitations.

Unlike in the integral quantum Hall regime, skyrmions at $\nu=\frac{1}{3}$ (proposed by Kamilla et al.47) were only recently detected in a transport experiment 48,49 thanks to a sufficient reduction of the Zeeman gap $E_{\mathrm{Z}}$ by means of hydrostatic pressure. The subsequent numerical calculation $\$ 0$ also indicated the formation of small skyrmions and anti-skyrmions in finite-size fractional quantum Hall systems. However, the question of why similar spin excitations occur in the $\nu=1$ electron system and the $\nu^{*}=1 \mathrm{fF}$ system despite different $e-e$ and $\mathrm{CF}-\mathrm{CF}$ interactions 2 has not yet been answered.

The situation in the excited LL's is not yet completely understood, either. First, it was predicted 51, 52 that skyrmions do not occur at $\nu=3$, 5, etc This prediction soon confirmed experimentally. 53 Then it was found 54 that a finite width of a quasi-2DEG stabilizes skyrmions in excited LL's. Indeed, rapid spin depolarization around $\nu=3$ was recently observed 55 in a rather wide, $30 \mathrm{~nm}$ quantum well. While it is clear that the finite width enters the problem of an isolated, excited LL only through the weakening of the $e-e$ repulsion at short range, the class of interactions for which skyrmions are stable has not yet been generally defined.

In this paper we compare the results of detailed numerical calculations of the spin excitation spectra at $\nu=\frac{1}{3}$, 1,3 , and 5 . We identify spin waves and skyrmions in each system, and analyze their single particle properties as well as mutual interactions. The spin excitation spectra turn out to be very similar at $\nu=\frac{1}{3}$ and 1 , and, if the layer width $w$ is sufficiently large, also at $\nu=3$ and 5. In particular, we confirm that skyrmions are the lowest-energy charged excitations in all these systems if the Zeeman energy $E_{\mathrm{Z}}$ is sufficiently small.

We show that the formation of particle-like skyrmions coincides with the occurrence of condensed states of a macroscopic number of nearly noninteracting chargeneutral spin waves each with angular momentum $L=1$. The interaction energy of these states is (nearly) linear in spin polarization $\zeta$ (and thus it remains finite at $\zeta=0$ ) giving rise to a gapless and continuous density of states. On the other hand, the correlations between charged (particle-like) skyrmions are expected to be of Laughlin type 4 meaning that the skyrmion pair eigenstates with the smallest relative pair angular momentum $\mathcal{R}$ are maximally avoided.50,24 26 This can be rephrased in terms of an effective spatial isolation of skyrmions from one another, and the absence of high-eneropskyrmionskyrmion collisions (at low temperature).565
We also determine a general criterion for the occurrence of skyrmions in a system of spin- $\frac{1}{2}$ particles halffilling a spin-degenerate shell (e.g., in a system of electrons or Laughlin QP's at $\nu=1$ and an arbitrary layer width $w$ ). We find that the particle-particle interaction pseudopotential ${ }^{5}(\mathcal{R})$ must: (i) be strongly repulsive (superharmonic24.25) at $\mathcal{R}=0$ to cause decoupling of the many-body states that avoid having $\mathcal{R}=0$ pairs from all other states (skyrmions are exact eigenstates of the ideal short-range repulsion with $V_{\text {ee }}(0)=\infty$ ), and (ii) decrease sufficiently quickly with increasing $\mathcal{R}$ between $\mathcal{R}=1$ and $3, \mathcal{R}=3$ and 5 , etc. to make the skyrmion energy decrease with increasing the topological charge $K$ (size) and, in particular, bring the skyrmion energy band below the energy of the spin-polarized QP state. For systems with broken particle $(\uparrow)-\operatorname{hole}(\downarrow)$ symmetry (e.g., at $\left.\nu=\frac{1}{3}\right)$, the latter condition must be rephrased in terms of the particle-hole pseudopotential $V_{\text {eh }}$ which (iii) must increase monotonically as a function of wave vector $k$.

The above criterion allows prediction and explanation of the occurrence or absence of skyrmions (and the resulting type of dependence of spin polarization $\zeta$ of the 2DEG on density or magnetic field) at an arbitrary filling factor $\nu$, layer width $w$, density profile across the layer $\varrho(z)$, etc. - based on the analysis of the involved $e-e$, $e-h, \mathrm{QE}-\mathrm{QE}, \mathrm{QH}-\mathrm{QH}$, or QE-QH interaction pseudopotentials. This criterion is somewhat analogous to the ope for Laughlin correlations in a partially filled shell Q, 2426 which explained compressibility of the spin-polarized $\nu=\frac{4}{11}$ state $\left(\mu=\frac{1}{3}\right.$ state of Laughlin QE's) and incompressibility 59 of the partially unpolarized state at the same $\nu=\frac{4}{11}\left(\nu=\frac{1}{3}\right.$ state of reversed-spin QE's 60.61 $)$.

\section{MODEL}

The model used here is essentially that of Ref. 59, except that in the present calculation we do not include valence-band holes. A finite system of $N$ electrons is confined on Haldane sphere 18 of radius $R$. The radial magnetic field $B$ is due to Dirac monopole, whose strength $2 Q$ is defined in units of flux quantum $\phi_{0}$, so that $4 \pi R^{2} B=2 Q \phi_{0}$ and $R^{2}=Q \lambda^{2}$. The single-electron states 18.62 , 63 are the eigenstates of magnitude and projection of angular momentum $(l$ and $m)$ and of the spin projection $(\sigma)$, and form $g$-fold $(g=2 l+1)$ degenerate LL's labeled by $n=l-Q=0,1,2, \ldots$.

The cyclotron energy $\hbar \omega_{c} \propto B$ is assumed much larger than the Coulomb interaction energy scale $E_{\mathrm{C}}=e^{2} / \lambda \propto$ $\sqrt{B}$, so that excitations between LL's can be neglected, and only one, isolated $n$th LL need be considered. On the other hand, no assumption is made about the Zeeman spin splitting $E_{\mathrm{Z}}$, which in GaAs samples can be made arbitrarily small by applying hydrostatic pressure. The ratio $\eta=E_{\mathrm{Z}} / E_{\mathrm{C}}$ of energy scales associated with spin and charge excitations (within an isolated LL) is a small free parameter of the model. 
Unless it is much larger than $\lambda$, finite layer width $w$ enters the problem by only modifying the quasi-2D interaction pseudopotential $V(\mathcal{R})$. In the lowest $\mathrm{LL}$, the effects due to a finite $w$ can be adequately modeled by merely reducing the Coulomb energy scale $E_{\mathrm{C}}$ by a factor $\xi_{w}<1$ compared to the ideal $w=0$ case $\left(\xi_{w} \sim \frac{1}{2}\right.$ in typical samples). However, the situation is very different in the excited LL's, where even if $w$ is sufficiently small that the inter-subband mixing can he neglected, it affects not only the overall energy scale 54 but the shape of $V(\mathcal{R})$, and thus the electron correlations 62426 as well. To address this problem, we have included finite $w$ in the calculation for excited LL's by using the quasi-2D Coulomb $e-e$ potential $V_{d}(r)=e^{2} / \sqrt{r^{2}+d^{2}}$, which is known 28.58 to reproduce well the pseudopotential $V(\mathcal{R})$ for $w \approx 5 d$. Here, $w$ is the effective layer width obtained from fitting the actual lowest-subband density profile $\varrho(z)$ with $\cos ^{2}(\pi z / w)$. In typical GaAs wells $w$ is larger than the well width by $3-3.5 \mathrm{~nm}$ due to a finite barrier height.

The Hamiltonian of interacting electrons confined to the $n$th LL can be written as

$$
H=\sum c_{m_{1} \sigma}^{\dagger} c_{m_{2} \sigma^{\prime}}^{\dagger} c_{m_{3} \sigma^{\prime}} c_{m_{4} \sigma}\left\langle m_{1} m_{2}|V| m_{3} m_{4}\right\rangle
$$

where $c_{m \sigma}^{\dagger}$ and $c_{m \sigma}$ are the electron creation and annihilation operators, and the interaction matrix elements are calculated for the potential $V_{d}(r)$ and they are connected with the pseudopotential $V(\mathcal{R})$ through the ClebschGordan coefficients. Hamiltonian $H$ is diagonalized in the basis of $N$-electron Slater determinants

$$
\left|m_{1} \sigma_{1} \ldots m_{N} \sigma_{N}\right\rangle=c_{m_{1} \sigma_{1}}^{\dagger} \ldots c_{m_{N} \sigma_{N}}^{\dagger}|\mathrm{vac}\rangle
$$

where $|v a c\rangle$ stands for the vacuum state. While using basis (2) allows automatic resolution of two good manybody quantum numbers, projection of spin $\left(S_{z}=\sum \sigma_{i}\right)$ and of angular momentum $\left(L_{z}=\sum m_{i}\right)$, the length of spin $(S)$ and of angular momentum $(L)$ are resolved numerically in the diagonalization of each $\left(S_{z}, L_{z}\right)$ Hilbert subspace. The results obtained on Haldane sphere are easily converted to the planar geometry, where $L$ and $L_{z}$ are appropriately 6465 replaced by the total and centerof-mass angular momentum projections, $M$ and $M_{\mathrm{CM}}$.

\section{INTEGRAL QUANTUM HALL REGIME (LOWEST LANDAU LEVEL)}

\section{A. Exactly filled level}

Let us begin the discussion of spin excitations of the 2DEG in the integral quantum Hall regime with the energy spectrum at precisely $\nu=1$. Similar spectra were previously stydied in the context of finite-size quantum Hall droplets.2 23 As an example, in Fig. 1(a) we show the finite-size spectrum for $N=12$ electrons on Haldane sphere with LL degeneracy $g=2 l+1=N$. In this and all other spectra in the paper, the excitation energy $E-E_{0}$

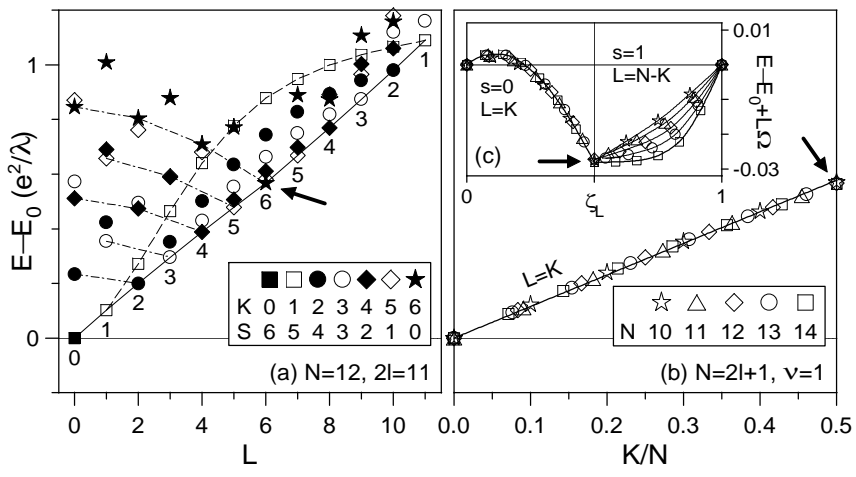

FIG. 1. (a) The energy spectrum (Coulomb energy $E$ versus angular momentum $L$ ) of the system of $N=12$ electrons in the lowest $(n=0)$ LL of degeneracy $g=2 l+1=12$ calculated on Haldane sphere. $S$ is the total spin, and $K=\frac{1}{2} N-S$ is the number of reversed spins. Dashed line: single spin wave; dash-dot lines: states containing equal numbers of $L=1$ spin waves; solid line: condensates of noninteracting $L=1$ spin waves. (b) The energy dispersion (energy $E$ versus spin polarization $\zeta=K / N$ ) for the spin-wave condensate states at $\nu=1$, calculated for $N \leq 14$ electrons exactly filling their lowest LL $(N=2 l+1)$. (c) The dispersion of (b), but with inclusion of a lateral confinement term $L \Omega$ and plotted as a function of normalized angular momentum $\zeta_{L} . \lambda$ is the magnetic length and arrows mark the same state in each frame.

is given in the units of $E_{\mathrm{C}}=e^{2} / \lambda$, measured from the energy $E_{0}$ of the lowest maximally spin-polarized state (here, the $\nu=1$ ground state), excludes the Zeeman energy $E_{\mathrm{Z}}$, and is plotted as a function of total angular momentum $L$ (for waves, related to longitudinal wave vector $k$ by $L=k R=k \lambda \sqrt{Q}$ ). Different symbols mark multiplets of different total spin $S$ and only the lowest state is shown at each $L$ and $S$. The labels show the number $K$ of reversed spins (relative to the maximally polarized state) defined as $K=\frac{1}{2} N^{\prime}-S$, where $N^{\prime}=\min (N, g-N)$.

It is well-known that even in the absence of the Zeeman energy gap $\left(E_{Z}=0\right)$ the ground state (GS) of the 2DEG at $\nu=1$ is spin-polarized (ferromagnetic) and translationally invariant (hence, nondegenerate). At the exact half-filling of a spin-degenerate LL, this GS is the only state with zero projection onto the most strongly repulsive $e-e$ pair eigenstate at $\mathcal{R}=0$. Indeed, Fig. 1 (a) shows a GS at $K=L=0$. Because of the complete filling of the spin- $\downarrow n=0$ LL in the ferromagnetic GS, the only excitations below the cyclotron gap $\hbar \omega_{c}$ are the spin waves (SW's) with $K=1$ and $L=1,2,3, \ldots$. A single SW consists of a vacancy (hole) in the spin$\downarrow$ level and an electron in the spin- $\uparrow$ level, and is alsa called a spin-exciton. Its dispersion curve is given by 66 $E_{\mathrm{SW}}(k)=E_{0}+E_{\mathrm{Z}}+E_{\mathrm{C}} \sqrt{\pi / 2}\left[1-\exp \left(-\kappa^{2}\right) I_{0}\left(\kappa^{2}\right)\right]$, where $\kappa=k \lambda / 2$ and $I_{0}$ is the modified Bessel function, and it is shown in Fig. 1(a) with a dashed line.

Interestingly, Fig. 1(a) shows that a single $\mathrm{SW}$ is not the lowest-energy excitation at $L>1$. Instead, the lowest excitations form a band with $K=L$ and energy nearly 
linear in $K$ (solid line). This (nearly) linear dependence $E(K)$ can be interpreted as the (nearly) perfect decoupling of SW's_each with $L=1$, earlier pointed out by Oaknin et al 42 . Denoting the energy of one SW by $\varepsilon_{\mathrm{SW}}$ allows us to approximate the lowest state with $K$ reversed spins by $E_{\mathrm{SW}}(K)=E_{0}+K \varepsilon_{\mathrm{SW}}$. Note that a pair of SW's each with $L=1$ can be in either parallel or anti-parallel state, with $K=2$ and pair angular momentum $L=2$ or 0 , respectively. It follows from Fig. 11(a) that the $L=2$ state containing a pair of SW's is noninteracting, while the $L=0$ state of such a pair is repulsive (the SW-SW repulsion energy at $L=0$ decreases as a function of system size, and for $N=12$ it is $\approx 0.03 E_{\mathrm{C}}$ ). Similarly, the states containing the number $K$ of SW's each with $L=1$ (see dotted lines) can have total angular momentum $L=K, K-2, K-4, \ldots$. However, only the $L=K$ state does not contain any SW-SW pairs with $L=0$, and thus only this state has energy $E=E_{\mathrm{SW}}(K)$, while all others have $E>E_{\mathrm{SW}}(K)$. The $L=K$ low-energy excitations of a 2DEG at $\nu=1$ are uniquely ordered states of noninteracting SW's each with $L=1$ (Bose-Einstein condensates) and will be denoted $W_{K}$.

The exact mapping 44 between the two-spin electron system $(\uparrow-\downarrow)$ and the electron-valence-hole $(e-h)$ system in the lowest LL allows the expression of the above statement in terms of the interaction between interband magneto-excitons; namely, the $L=1$ excitons in the lowest LL do not interact with one another and they condense into correlated $L=K$ states. Although similar, this symmetry js independent from the well-known "hidden symmetry" 67 and the decoupling of $L=0$ excitons 44565 Its consequence will be also different because, in contrast to the radiative $L=0$ excitons, the $L=1$ excitons are long-lived.

To determine the energy spectrum of an infinite 2DEG we have compared data obtained for different electron numbers, $N \leq 14$. As shown in Fig. 目(b), it turns out that the energy $E_{W}$ of $W_{K}$ excitations is an almost linear function of the "relative" spin polarization, $\zeta=K / N$. Namely, $E_{W}=E_{0}+u \zeta$, with the slope that we estimate as $u \approx 1.15 E_{\mathrm{C}}$. This fact reflects the extended character of the SW's and has a couple of obvious consequences for the thermodynamic limit of $N \rightarrow \infty$ : (i) For any $E_{\mathrm{Z}} \neq 0$, the interaction energy $E_{W}(K)-E_{0} \propto K / N$ of each correlated $W_{K}$ state is negligible compared to its total Zeeman energy, $K E_{\mathrm{Z}}$. (ii) The gap for spin excitations is precisely the Zeeman gap $E_{\mathrm{Z}}$; if this gap can be closed (e.g., by applying hydrostatic pressure), the $\nu=1$ ferromagnetic GS becomes gapless, the density of states for the $W_{K}$ excitations becomes continuous, and a macroscopic number $\sim N k_{\mathrm{B}} T / u$ of noninteracting SW's occur at an arbitrarily small finite temperature.

To remove the dominant linear term from $E_{W}(\zeta)$ and study the small nonlinear correction, in Fig. 11(c) we plot the energy spectrum shifted by an additional term $L \Omega$. Physically, this term describes a harmonic lateral confinement applied to a finite-size $\nu=1$ droplet 4143 The confinement strength $\Omega$ is chosen so that the $\nu=1$ GS is degenerate with the next "compact droplet" state at $K=1$ and $L=N-1$ (compact-droplet eigenstates 414 $C_{K}$ with $K \geq 1$ are created by $\prod_{i=0}^{K-1} c_{l-i, \uparrow}^{\dagger} c_{-l+i \downarrow}$ acting on $C_{0}=\prod_{m=-l}^{l} c_{m, \downarrow}^{\dagger}|\mathrm{vac}\rangle$, i.e. on the $\nu=1 \mathrm{GS}$ ). To compare data for different $N$, the energy is plotted as a function of normalized angular momentum, $\zeta_{L}=\left(L-s^{2}\right) /(N-2 s)$, where $s$ is equal to $K$ of the nearest $C_{K}$ state, so that $\zeta_{L}$ increases with $L$ and equals $K$ in each $C_{K}$ state (note that $\zeta_{L}=\zeta$ for $\zeta_{L} \leq \frac{1}{2}$ ).

Clearly, the nonlinear correction to $E_{W}-E_{0}$ is also a well-defined function of $\zeta$ or $\zeta_{L}$ with a minimum at $\zeta_{L}=\frac{1}{2}$, i.e. in the highly correlated singlet $(S=0)$ state marked with arrows. If both $E_{\mathrm{Z}}$ and $N$ are sufficiently small, the energy of this singlet state, $E_{W}\left(\frac{1}{2}\right)-E_{0}+$ $\frac{1}{2} N \Omega \approx-0.03 E_{\mathrm{C}}+\frac{1}{2} N E_{\mathrm{Z}}$ can remain negative. Then, the sublinear behavior of $E\left(\zeta_{L}\right)$ between $\zeta_{L}=0$ and $\frac{1}{2}$ implies an abrupt transition from the $\nu=1$ ferromagnetic GS to the $\zeta_{L}=\frac{1}{2}$ singlet GS (skipping intermedi-

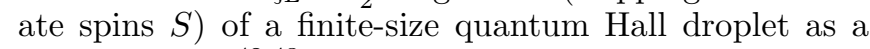
function of $\Omega .42 .33$ On the other hand, the superlinear behavior of $E\left(\zeta_{L}\right)$ between $\zeta_{L}=\frac{1}{2}$ and 1 implies that a further decrease of $\Omega$ drives a quantum Hall droplet from the $S=0$ state to the $S=N-1$ compact state through a series of GS's with all intermediate spins.

\section{B. Additional particle or hole in filled level}

What truly ignites the abrupt depolarization of a finite $\nu=1$ droplet under the variation of the lateral confinement is the insertion of an additional reversed-spin particle (from the edge) into the bulk of the droplet. This effect is a consequence of a more general phenomenon the occurrence of particle-like chargef excitations with macroscopic spin called skyrmions 1517 Let us consider a (possibly infinite) system of spin- $\frac{1}{2}$ fermions half-filling $(\nu=1)$ a degenerate $\left(E_{\mathrm{Z}}=0\right)$ shell of single-particle states and forming a ferromagnetic GS in accordance with a standard atomic Hund's rule. Depending on details of the interaction pseudopotential, addition of a single particle or hole to such system may or may not cause its complete depolarization, that is a transition to a highly correlated singlet GS in which half of the electrons flipped their spins. Examples of systems in which such depolarization does or does not occur are electrons in the lowest LL or in an atomic shell, respectively.

The effect of removing an electron from a $\nu=1$ GS is presented in Fig. 2(a), showing the energy spectrum of $N=12$ electrons at $2 l=12$ (i.e., $N$ smaller by one than the LL degeneracy $g=2 l+1$ ). Similar spectra were first analyzed by Xie and He 34 Because of the exact particle-hole symmetry in the lowest LL, this system can be viewed as containing either one hole or one extra electron in a $\nu=1$ GS of 13 electrons, and will be labeled as $\nu=1^{ \pm}$. Clearly, while the right-hand part of the spectrum (the single SW and $L=N-S$ bands at $L \geq \frac{1}{2} N$ ) resembles that of Fig. 1 (a), a new band of states with 

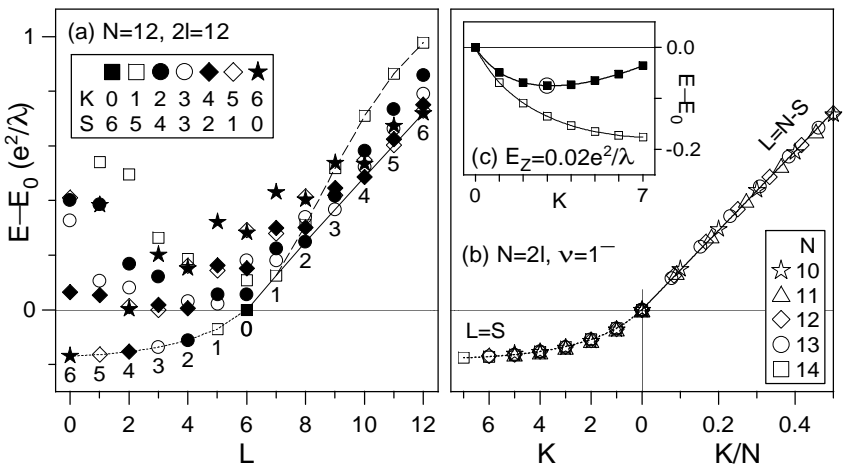

FIG. 2. (a) Same as Fig. 11(a) but for $g=2 l+1=13$. Dashed line: single spin wave in the presence of a hole; solid line: spin-wave condensates in the presence of a hole; dotted line: skyrmions. (b) Same as Fig. 1(b) but for $N=2 l$, i.e. one hole in the $\nu=1$ state. The skyrmion and spin-wave condensate energies are plotted as a function of numbered of reversed spins $K$ and spin polarization $\zeta=K / N$, respectively. (c) Open and full symbols: skyrmion dispersion of (b) for $N=14$ without and with Zeeman energy $K E_{\mathrm{Z}}$.

$L=S$ appears at $0 \leq L<\frac{1}{2} N$, at the energy below the lowest $K=0$ state. These are the skyrmion states of topological charge $K$, denoted here by $S_{K}$ and first identified in nuclear physics by Skyrme 15 and in the fractional quantum Hall systems by Sondhi et al 29 In these states, a number $K=1,2, \ldots$ of SW's (spin-excitons) binds to a hole (strictly speaking, skyrmions consist of SW's bound to an electron, and the particle-hole symmetric state consisting of SW's and a hole is called apanti-skyrmion). In the $e^{-} h$ picture the $S_{K}$ states map 4 onto the charged multi-excitons $19.46 X_{K}^{ \pm}$, consisting of $K e-h$ pairs bound to an extra $e$ or $h$.

To understand the energy spectrum of an infinite 2DEG at $\nu=1^{ \pm}$, in Fig. 2(b) we compare data obtained for different $N$. Similarly to the $L=K$ band at $\nu=1$, the energy of $L=N-S$ states at $\nu=1^{ \pm}$turns out to be a nearly linear function of the spin polarization, $E_{W}^{\prime}=E_{0}+u^{\prime} \zeta$, only with a slightly increased slope, $u^{\prime}=u+v$ with $v \approx 0.3 E_{\mathrm{C}}$. Since the angular momentum $L=N-S$ can be obtained by adding $L=K$ for the $W_{K}$ condensate and $l=\frac{1}{2} N$ for the single hole (assuming their parallel orientation), energy $v / N$ can be interpreted as the repulsion between a finite-size hole and (oriented "parallel" to it) one extended SW.

In view of their charged multi-exciton interpretation in the $e-h$ picture, it is not surprising that the relevant quantum number to label the $L=S$ skyrmion excitations $S_{K}$ in an infinite system is $K$ (and not $\zeta=K / N$ appropriate for $W_{K}$ condensates). Indeed, Fig. 2(b) shows that the excitation energy $E_{S}-E_{0}$ of the $S_{K}$ states is a function of $K$ (rather than of $\zeta$ ), with the discrete series of values of $E_{S}(K)$ quickly converging as $N$ is increased (careful extrapolation to $N \rightarrow \infty$ gives $E_{S}(K)-E_{0}=-0.0529,-0.0828,-0.1018, \ldots$, $-\frac{1}{4} \sqrt{\pi / 2}=-0.3133$ for $K=1,2,3, \ldots, \infty$; all in units of $\left.E_{\mathrm{C}}\right)$. The fact that $E_{S}(K)-E_{0}<0$ means that in the $S_{K}$ state the attraction between the hole (or electron) and $K$ SW's overcomes the creation energy of these SW's, and the ferromagnetic state with $K=0$ may become unstable. Most importantly, as shown in Fig. 2(c), if $E_{\mathrm{Z}}$ is nonzero but smaller than $E_{0}-E_{S}(K)$, then regardless of its actual value a particle-like GS will occur with an excitation gap that is much smaller than the gap at precisely $\nu=1$ (which is $E_{\mathrm{Z}}$ ). In other words, introduction of additional electrons (or holes) to the incompressible $\nu=1$ GS with a gap $E_{\mathrm{Z}}$ will cause significant reduction of the gap for spin excitations, and the objects that are able to reverse spin at low energy (much below $E_{\mathrm{Z}}$ ) are finite-size, charged particles (skyrmions) that move in the underlying $\nu=1$ fluid on electron-like cyclotron orbits. The ability of (mobile) skyrmions to increase and decrease spin at an energy cost that is small compared to and largely independent of $E_{\mathrm{Z}}$ (all in contrast to the $\nu=1$ state) causes critical magnetic field dependence of the spin relaxation rate for magnetic particles interacting with the 2DEG, such as ions, nuclei, or charged excitons.

While the decoupling of SW's follows from the linear dependence of $E_{W}$ on $K$ (or $E_{W}^{\prime}$ on $K$, for the SW's in the presence of an extra electron or a hole), the nature of interaction between skyrmions follows froman earlier study of the $e-h$ complexes. It was shown 56.57 that the interaction between any pair of charged excitons $X_{K}^{ \pm}\left(X_{0}^{ \pm}\right.$means an electron or a hole) with equal charge $( \pm e)$ but possibly different sizes $\left(K \neq K^{\prime}\right)$ is repulsive and similar to the $e-e$ interaction. In particular, all $K-K^{\prime}$ repulsion pseudopotentials (defined 5 as the pair interaction energy as a function gf pair angular momentum) have short range, implying 25 that an $X_{K}^{ \pm}$particle will have Laughlin correlations $]$ with all other $X_{K^{\prime}}^{ \pm}$particles in the system. Laughlin correlations are described by an appropriate Jastrow prefactor in the many-body wave function and mean the tendency to avoid the pair states with maximum repulsion (minimum average separation). This implies that (at low temperature) an $X_{K}^{-}$ does not undergo high-energy collisions with any other $X_{K^{\prime}}^{-}$charges. Since the same must hold for skyrmions, we conclude that regardless of their size $(K)$ or density, the skyrmions will be effectively isolated from one another. This makes finite size skyrmions well-defined quasiparticles, virtually unperturbed by the skyrmion-skyrmion scattering, and excludes many-skyrmion effects from a possible spin coupling of a $2 \mathrm{DEG}$ to magnetic particles.

\section{FRACTIONAL QUANTUM HALL REGIME}

Let us nowfollowing Kamilla et al. $\mathbf{t}^{-}$and MacDonald and Palacios, 0 turn to the question if the spin excitations analogous to the spin-wave condensates and $S_{K}$ skyrmion particles described in the preceding section might also occur in the fractional quantum Hall regime. On one hand, it is known that Laughlin correlations $\$$ in an electron sys- 


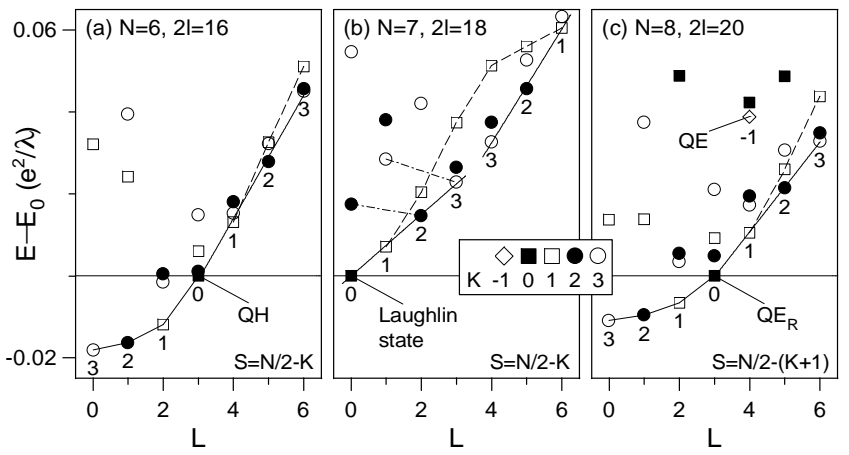

FIG. 3. Same as Figs. 1(a) and B(a) but for the LL degeneracy $g=2 l+1$ equal (b), greater by one (a), or smaller by one (c) to/from the value corresponding to $\nu=\frac{1}{3}$.

tem near $\bar{C}(2 p+1)^{-1}$ (where $p$ is an integer) allow the mapping 1921 of the low-energy states onto the noninteracting $\mathrm{CF}$ states with an effective filing factor $\nu^{*} \approx 1$. This mapping is done by replacing the electron LL degeneracy $g$ by $g^{*}=g-2 p(N-1)$, which can be interpreted as attachment of $2 p$ magnetic flux quanta to each electron. On a sphere, 6 this replaces $2 l=2 Q \approx(2 p+1)(N-1)$ by $2 l^{*}=2 Q^{*} \approx N-1$. On the other hand, it is the specific form of the interactions between the reversed-spin electrons and holes at $\nu=1$ that causes occurrence of $W_{K}$ and $S_{K}$ excitations, and the interaction between these excitations in an electron system is quite different from the residual interaction between CF's. A well-known example demonstrating that the analogy between the electron and CF systems does not always work because of different interactions is the postulate of similar Laughlin correlations at the $\nu=(2 p+1)^{-1}$ fillings of electron or CF LL's, giving rise to Haldane hierarchy of incompressible fractional quantum Hall states. 18 For example, the $\nu=\frac{1}{3}$ states of the vacancies in the spin- $\downarrow n=0$ CF LL (Laughlin QH's) and of particles in the spin- $\downarrow$ $n=1$ CF LL (Laughlin QE's) or in the spin- $\uparrow n=0 \mathrm{CF}$ LL (reversed-spin quasielectrons, 60 , $61 \mathrm{QE}_{\mathrm{R}}$ ) correspond to the polarized strongly incompressible $\nu=\frac{2}{5}$ and compressible $\nu=\frac{4}{11}$ states 27 and to the partially unpolarized weakly incompressible $59 \nu=\frac{4}{11}$ state, respectively.

The examples of energy spectra at $\nu \approx \frac{1}{3}$ are shown in Fig. 3. The values of $N$ and $2 l$ are chosen so that $g^{*}=7$ in each frame and the "reference" state with $K=0$ and $E=E_{0}$ is one $\mathrm{QH}\left(g^{*}=N+1\right.$ and $\left.\nu^{*}=1^{-}\right)$, Laughlin state $\left(g^{*}=N\right.$ and $\left.\nu^{*}=1\right)$, and one $\mathrm{QE}_{\mathrm{R}}\left(g^{*}=N-1\right.$ and $\left.\nu^{*}=1^{+}\right)$in Figs. $3(\mathrm{a}),(\mathrm{b})$, and (c), respectively. Some of the energies for smaller values of $N$ and/or $K$ have been recently obtained by MacDonald and Palacios. 50

Clearly, the SW dispersion $E_{\mathrm{SW}}(K)$, the linear $E_{W}(K)$ band, as well as the $E_{S}(K)<0$ band are all present in the spectra, in analogy to Figs. 1(a) and 2(a). What is visibly different from the $\nu=1$ spectra is a smaller energy scale (predominantly due to a fractional charge of involved $\mathrm{QH}, \mathrm{QE}$, and $\mathrm{QE}_{\mathrm{R}}$ quasiparticles), and a discrepancy between the $\nu=1^{-}$and $1^{+}$spectra. The latter

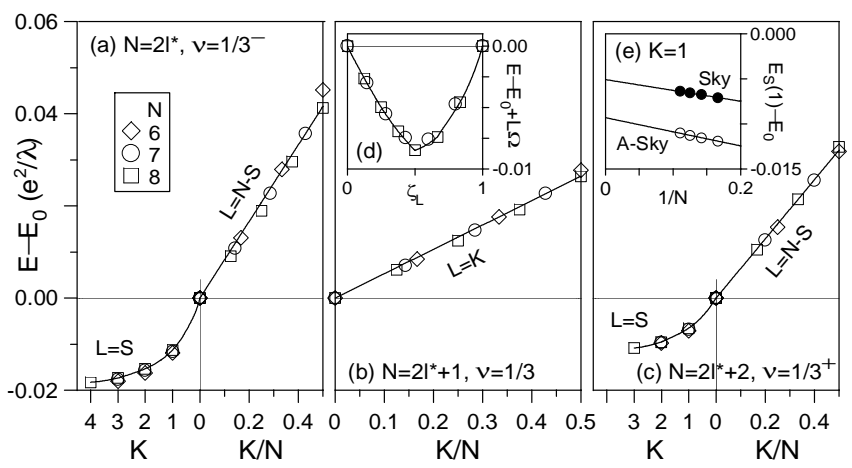

FIG. 4. Same as Figs. 1 (b) and R(b) but for the LL degeneracy $g=2 l+1$ equal (b), greater by one (a), or smaller by one (c) to/from the value corresponding to $\nu=\frac{1}{3}$. Insets: (d) Same as Fig. 11(c) but for $\nu=\frac{1}{3}$. (e) Energies $E_{S}(1)$ of skyrmions (at $\nu=\frac{1}{3}^{+}$) and anti-skyrmions (at $\nu=\frac{1}{3}^{-}$) with $K=1$ as a function of inverse electron number.

reveals only approximate particle-hole symmetry within the lowest CF LL (it was shown earlier 5 that the $\mathrm{QH}-$ $\mathrm{QH}$ and $\mathrm{QE}_{\mathrm{R}}-\mathrm{QE}_{\mathrm{R}}$ interactions are quite different). This implies broken symmetry between the skyrmion and antiskyrmion states 50 in contrast to the $\nu=1$ case.

Let us analyze the spectra in Fig. 3 in more detail. The charge excitations of the Laughlin $\nu=\frac{1}{3}$ GS in Fig. 3(b) are all above the magneto-roton gap $\left(E-E_{0} \approx 0.081 E_{\mathrm{C}}\right.$ for $N=7)$ and are not shown. The spin excitations include a single SW (an excitonic bound state of a $\mathrm{QH}-$ $\mathrm{QE}_{\mathrm{R}}$ pair) marked by a dashed line. Below a single SW, there is a linear band of $W_{K}$ states with $L=K$ which, in analogy to the $\nu=1$ spectra, can be interpreted as Bose condensates of $K$ noninteracting SW's each with $L=1$ and all having parallel angular momenta. As shown in Fig. 1(b), similarly as at $\nu=1$, the energy of $W_{K}$ states is a nearly linear function of $\zeta$, $E_{W}=E_{0}+u \zeta$, with $u=0.053 E_{\mathrm{C}}$ (much less than $\frac{1}{9}$ times the value for $\nu=1$, that would only take into account the smaller charge of $\pm \frac{1}{3} e$ for QH's and $\mathrm{QE}_{\mathrm{R}}$ 's). As at $\nu=1, E_{W}(K)-E_{0} \propto K / N$ means no gap and a continuous density of states if the Zeeman gap can be closed. The comparison of Figs. A(e) and If(c) shows that applying lateral confinement can also force the spin-polarized finite-size $\nu=\frac{1}{3}$ fractional quantum Hall droplet to undergo transition to the spin-singlet $(S=0)$ correlated GS at $L=\frac{1}{2} N$, just as it was at $\nu=1$.

The linear bands at $L=N-S$ found in Fig. 2 occur also at $\nu=\frac{1}{3}^{ \pm}$in Figs. 3(a) and (c). By analogy, these states correspond to a number $K$ of SW's each with $L=1$, coherently created in the presence of a $\mathrm{QH}$ or $\mathrm{QE}_{\mathrm{R}}$. The $\mathrm{QH}-\mathrm{SW}$ and $\mathrm{QE}_{\mathrm{R}}-\mathrm{SW}$ interaction constants $v$, obtained from the $E_{W}^{\prime}=E_{0}+(u+v) \zeta$ fits as shown

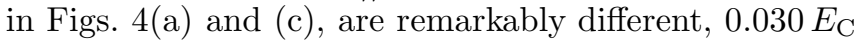
and $0.011 E_{\mathrm{C}}$, respectively.

The discrete skyrmion and anti-skyrmion bands at $L=$ $S$ in Figs. 3(a) and (c) also resemble their $\nu=1$ counterpart in Fig. 1 (a), and the energies $E_{S}(K)$ all seem to con- 
verge when $N \rightarrow \infty$. For example, in Fig. 国(e) the linear extrapolation of the energies of skyrmions (Sky) and antiskyrmions (A-Sky) with $K=1$, obtained for $N \leq 9$, gives $E_{S}(1)-E_{0}=-0.0050 E_{\mathrm{C}}$ and $-0.0093 E_{\mathrm{C}}$, respectively (compare with $-0.0529 E_{\mathrm{C}}$ for $\nu=1$ ). These are the critical values of the Zeeman energy $E_{\mathrm{Z}}$, below which these excitations can be observed experimentally. Note also that the $K=1$ skyrmion and anti-skyrmion states are perfect analogs of the interband charged exciton states, except that $e$ and $h$ are replaced by $\mathrm{QE}_{\mathrm{R}}$ and $\mathrm{QH}$. In analogy to $\nu=1$, the values of $E_{S}(1)-E_{0}$ measure the binding energies of such (fractionally) charged $\mathrm{CF}$ spinexcitons, $X_{\mathrm{CF}}^{-}=2 \mathrm{QE}_{\mathrm{R}}+\mathrm{QH}$ and $X_{\mathrm{CF}}^{+}=2 \mathrm{QH}+\mathrm{QE}_{\mathrm{R}}$.

It might seem surprising that the spin excitations at $\nu=1$ and $\frac{1}{3}$ are similar despite different interactions between electrons and CF's. However, of all three types of QP's at $\nu=\frac{1}{3}$, only $\mathrm{QH}$ and $\mathrm{QE}_{\mathrm{R}}$ are involved in the low-energy spin excitations $W_{K}$ and $S_{K}$, and the $\mathrm{QH}-$ $\mathrm{QH}, \mathrm{QH}-\mathrm{QE}_{\mathrm{R}}$, and $\mathrm{QF}_{R}-\mathrm{QE}_{\mathrm{R}}$ pseudopotentials describing their interactions, ${ }^{2} \Phi$ are all quite similar to the $e-e$ and $e^{-} h$ pseudopotentials in the lowest electron LL. On the other hand, the QE's, whose interaction (at short range) with one another and with other QP's is very different,27 do not participate in $W_{K}$ and $S_{K}$ excitations.

\section{INTEGRAL QUANTUM HALL REGIME (EXCITED LANDAU LEVELS)}

Another system with an identical structure of the single-particle Hilbert space but with different interactions is a nearly completely filled excited $(n>0)$ LL, experimentally realized in the 2DEG at $\nu \approx 2 n+1$. Note that if skyrmions would indeed occur at $\nu=3$, 5 , etc., they should be observed even more easily than at $\nu=1$ because of the weaker magnetic field $B$ (at the same 2DEG density), and thus smaller $\eta=E_{\mathrm{Z}} / E_{\mathrm{C}} \propto \sqrt{B}$.

In this section we shall discuss the results for an ideal $2 \mathrm{D}$ system with zero layer width, $w=0$. The energy spectra analogous to those in Figs. 1(a) and 2(a) but calculated for $n=1$ and 2 are shown in Figs. 5 and 6 .

The same the number of electrons $N=12$ (in the $n \mathrm{th}$ LL; the lower LL's are completely filled) and the angular momenta $l=11$ and 12 have been chosen, yielding the monopole strength $2 Q=2(l-n)$. Clearly, none of the above-discussed features of the $\nu=1$ or $n=\frac{1}{3}\left(\nu^{*}=1\right)$ spectra are present at $\nu=3$ or 5 .

Let us begin with Fig. 5 for $N=g$. A single SW (dashed lines; for dispersion see Ref. 66) is generally the lowest-energy spin excitation (at any $L$ ), and the $W_{K}$ bands (identified by comparison of the pair correlation functions) have higher energy and are no longer linear. The sublinear and nearly parabolic $E_{W} \approx E_{0}+u \zeta+y \zeta^{2}$ can be interpreted as an attraction between the $L=1$ SW's and, based on data for $N \leq 14$, we find $u=2.41 E_{\mathrm{C}}$ and $y=-1.62 E_{\mathrm{C}}$ for $n=1$, and $u=3.45 E_{\mathrm{C}}$ and $y=-2.88 E_{\mathrm{C}}$ for $n=2$. In a finite-size $\nu=3$ or 5

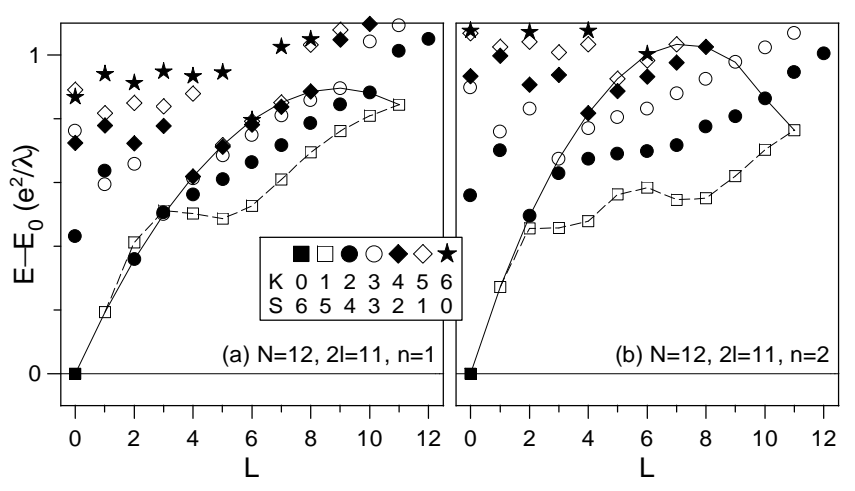

FIG. 5. Same as Fig. 11(a) but for electrons confined to the first (a) and second (b) excited LL.

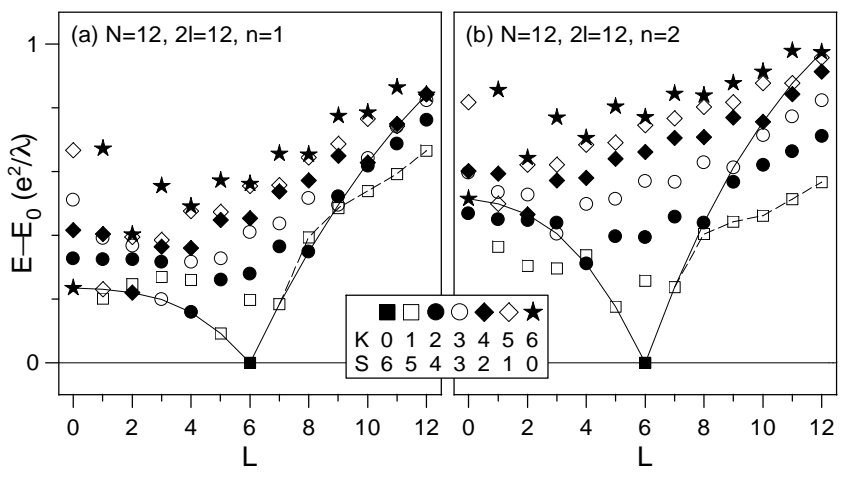

FIG. 6. Same as Fig. 2(a) but for electrons confined to the first (a) and second (b) excited LL.

droplet, as a result of this attraction, the spin-singlet condensate of $K=\frac{1}{2} N$ SW's (marked with arrows in Fig. 1) is an excited state at any strength of confinement $(\Omega)$, and the edge-reconstruction of the $\nu=3$ or 5 ferromagnetic GS $\left(C_{0}\right)$ occurs directly to the next compact-droplet state, $C_{1}$. This different behavior might be probed in a transport experiment by sending a reversed-spin electron over a quantum dot containing a compact droplet. It seems that a reversed-spin carrier would induce and bind SW's when sent through a $\nu=1$ or $\frac{1}{3}$ droplet, and travel ballistically through a $\nu=3$ or 5 droplet.

The lack of response to an addition of a reversed-spin electron (from the edge into the inside of the droplet) must mean unbinding of skyrmions at $\nu=3$ or 5 . Indeed, the $S_{K}$ states in the spectra for $N+1=g$ in Fig. 6 all have $E>E_{0}$. This means no skyrmions in the excited LL's at any value of $E_{\mathrm{Z}}$. In contrast to the situation near $\nu=1$ or $\frac{1}{3}$, the GS both precisely at $\nu=3$ or 5 and in the vicinity of these values remains maximally spin-polarized even in the absence of Zeeman splitting. In the $e-h$ picture, the result is that no bound chargedexciton states $X_{K}^{-}$occur in excited LL's (in the absence of inter-LL mixing and finite well width effects). 


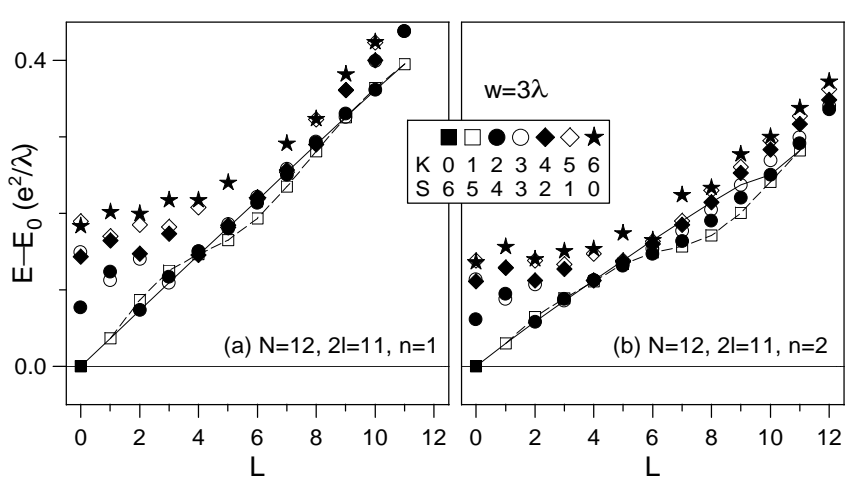

FIG. 7. Same as Fig. 5 but for a finite width $w=3 \lambda$ of a quasi-2D electron layer.

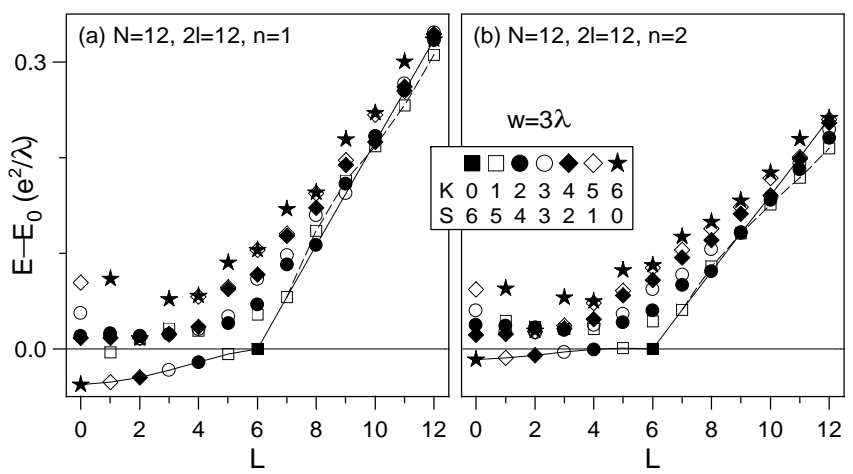

FIG. 8. Same as Fig. 6 but for a finite width $w=3 \lambda$ of a quasi-2D electron layer.

\section{EFFECTS OF FINITE LAYER WIDTH}

As was first predicted by Cogper 54 and later confirmed experimentally by Song et al.,5 skyrmions become the lowest-energy charged excitations in the excited LL's as well, if only the layer width $w$ is sufficiently large. We have calculated the spin-excitation spectra analogous to those of Figs. 5 and 6 but for $w=3 \lambda$, and show them in Figs. 17 and 8. For the exact fillings of the $n=1$ and 2 LL's $\left(\nu=3\right.$ and 5; Fig. 17), the $E_{W}(K)$ energy bands which were strongly sublinear for $w=0$ now become nearly linear (similar to the lowest LL; see Fig. 1). This indicates vanishing of the SW-SW attraction, and reoccurrence of the Bose condensate of ordered $L=1$ SW's. For an additional hole in the $n=1$ and 2 LL's $\left(\nu=3^{-}\right.$ and $5^{-}$; Fig. 8), the skyrmion energy bands which had $E_{S}(K)>E_{0}$ for $w=0$ now have $E_{S}(K)<E_{0}$ (again, similar to the lowest LL; see Fig. 2). This indicates stability of skyrmions in excited LL's in a wide quasi-2D layer (at sufficiently small $E_{\mathrm{Z}}$ ). Also in Fig. 8, the $L=N-S$ bands of states (containing $K$ SW's created coherently in the presence of a hole) become now nearly linear, in contrast to the behavior in Fig. 6 but similarly to Fig. 2 .

In Fig. 9 we compare the energies of skyrmions with $K=1,2$, and $\frac{1}{2} N$ (the latter state has $S=0$ and would correspond to an infinite-size skyrmion in the $N=\infty$

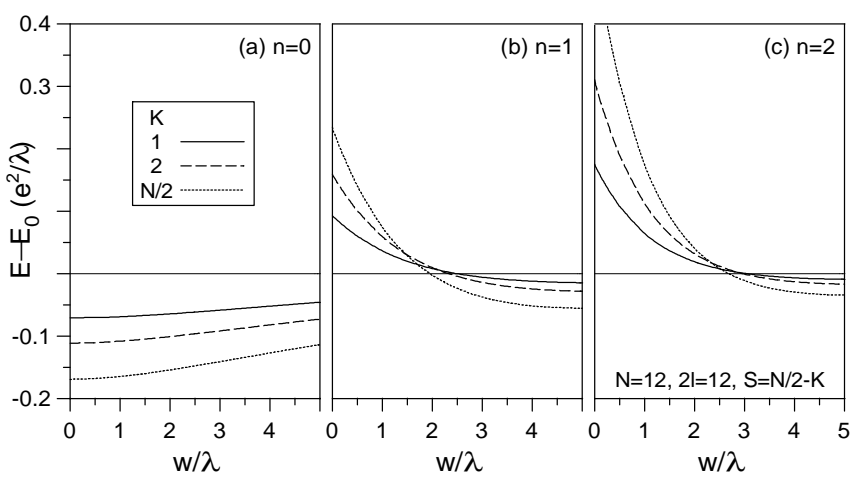

FIG. 9. The energy $E$ of skyrmions with $K=1,2$, and $\frac{1}{2} N$ as a function of the layer width $w$, calculated on Haldane sphere for $N=12$ electrons in the lowest (a), first excited (b), and second excited (c) LL. $\lambda$ is the magnetic length.

limit) plotted as a function of the layer width $w$. Clearly, the skyrmion energy is more sensitive to $w$ in the excited LL's. The "binding energies" $E_{S}(K)-E_{0}$ that were all positive for $w=0$ in Fig. 6 change sign at $w / \lambda=2$ to 3 , depending on $K$ and $n$. Note that our critical values of $w$ are quite higher than those predicted by Cooper (for example, for the $n=1 \mathrm{LL}$, his critical parameter $a=0.09 \lambda$ for the gaussian density profile, $\varrho(z) \propto \exp \left(-z^{2} / 2 a^{2}\right)$ corresponds to $w \approx 0.5 \lambda$ for our $\left.\varrho(z) \propto \cos ^{2}(z \pi / w)\right)$. This discrepancy indicates slow convergence of the energy of an infinite $(S=0)$ skyrmion with the electron number $N$. However, our critical values are certainly more appropriate for small skyrmions which are observed experimentally. Let us compare these values with a pair of experiments in which the skyrmions were and were not observed at $\nu=3$. Taking parameters after Song et al. 55 who observed skyrmions with $K \leq 2(B=2.15 \mathrm{~T}$ and well width of $30 \mathrm{~nm}$ yielding $w=33.5 \mathrm{~nm}$ ) gives $w / \lambda=1.9$, just above our critical value (see Fig. 11 for data for $N \rightarrow \infty$ ). On the other hand, taking the parameters after Schmeller et al. 53 who did not observe skyrmions $(B=2.3 \mathrm{~T}$ and well widths of 14 and $20 \mathrm{~nm}$ yielding $w=17.5$ and $23.5 \mathrm{~nm}$ ) gives $w / \lambda=1.03$ and 1.40 for two different samples, both significantly below our critical value and above that given by Cooper.

Using the data of Fig. 9 one can calculate a phase diagram for the occurrence of skyrmions with a given number of reversed spins $K$ as a function of the layer width $w$ and the Zeeman energy $E_{\mathrm{Z}}$. Such a diagram is presented in Fig. 10 for the integral filling of the lowest and first two excited LL's ( $\nu=1,3$, and 5 , respectively). To have a more reliable estimate of the critical $w / \lambda$ and $\eta$ for the occurrence of skyrmions with any $K \leq 1$, we have recalculated the curves for $K=1$ and 2 for much larger $N$ (up to 50) and then, thanks to their regular dependence on $N$, were able to extrapolate them to the $N \rightarrow \infty$ limit. The resulting phase diagram, shown in Fig. 11, describes an infinite planar system, and it is consistent with the skyrmion energies reported by Palacios et al.16 for $n=0$ and $w=0$. Remarkably, the critical value 


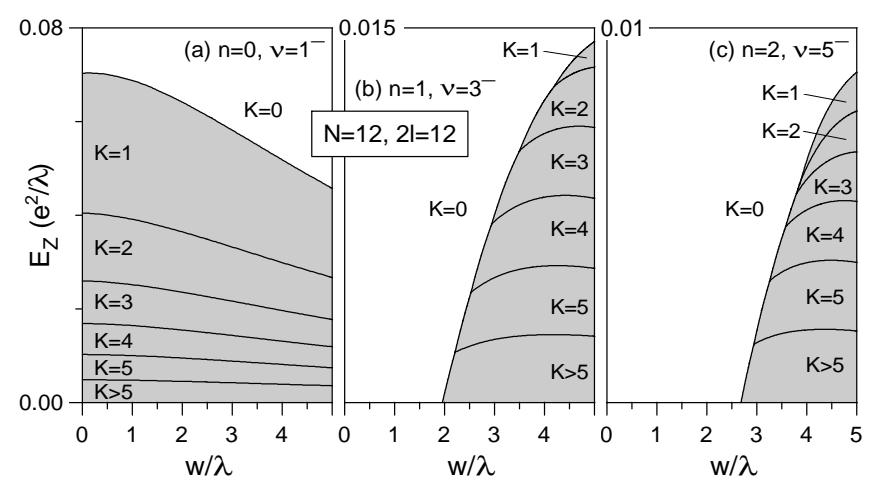

FIG. 10. Phase diagrams for the occurrence of skyrmions with $K$ reversed spins in a quasi-2D electron gas of finite width $w$, calculated in the system of $N=12$ electrons in the lowest (a), first excited (b), and second excited (c) LL of degeneracy $g=2 l+1=12$. $E_{\mathrm{Z}}$ is the Zeeman energy and $\lambda$ is the magnetic length. The numbers in the top-left corners of frames (b) and (c) give the upper bounds of their vertical axes (the lower bound are zero in all frames).

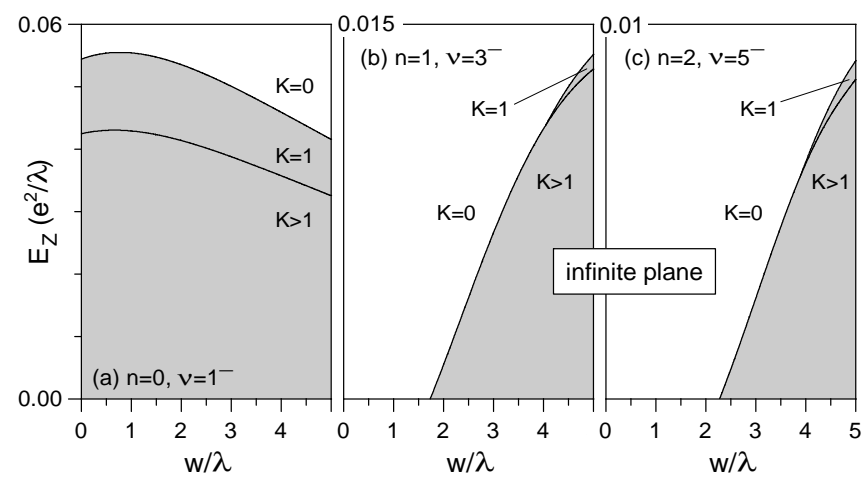

FIG. 11. Same as Fig. 10 but for an infinite planar system (critical values of Zeeman energy $E_{\mathrm{Z}}$ for each layer width $w$ were obtained from extrapolation of data for electron numbers $N \leq 50$ to the limit of $N \rightarrow \infty$ ).

of $E_{\mathrm{Z}}$ for the lowest LL is quite insensitive to $w$ over a wide range of layer widths. This is in contrast to the situation in the excited LL's, for which the phase diagrams in Fig. 11(bc) show a similar fast increase of the critical $E_{\mathrm{Z}}$ with increasing $w$. The critical layer widths in the limit of vanishing Zeeman energy are $w / \lambda=1.8$ and 2.3 for $n=1$ and 2 , respectively. In view of a recent study 20 that showed that the LL mixing only weakly affects the skyrmion energies in the layers of nonzero width, we expect our phase diagrams in Fig. 11 to be quite adequate for realistic experimental systems.

Finally, in Fig. 12 we present an analogous phase diagram for the $\nu=\frac{1}{3}$ fractional quantum Hall state. Due to the broken $\mathrm{QE}_{\mathrm{R}}-\mathrm{QH}$ symmetry, the diagrams for skyrmions (at $\nu=\frac{1}{3}^{+}$) and anti-skyrmions (at $\nu=\frac{1}{3}^{-}$) are different, and they are both shown. The solid lines and shaded areas give the result for small systems: $N=7$ and $2 l=19$ in frame (a), and $N=8$ and $2 l=20$ in frame (b). The dashed lines give the critical values of $E_{\mathrm{Z}}$

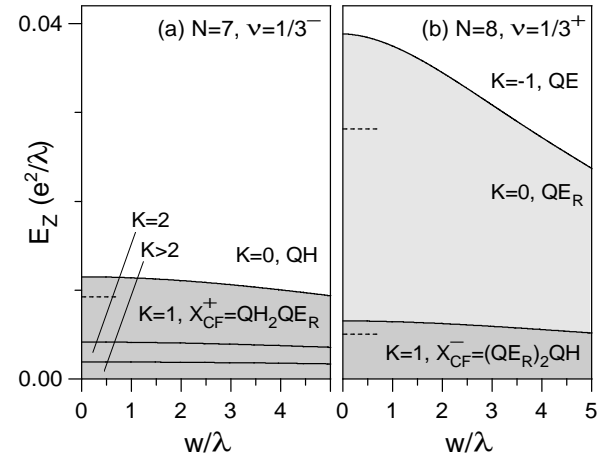

FIG. 12. Same as Fig. 10 but for the fractional quantum Hall states near $\nu=\frac{1}{3}$. Horizontal dashes lines mark the critical values of $E_{\mathrm{Z}}$ at $w=0$ obtained from extrapolation of the finite-size data for $N \leq 9$ to the $N \rightarrow \infty$ limit.

at $w=0$ for the $X_{\mathrm{CF}}^{+}, \mathrm{QE}_{\mathrm{R}}$, and $X_{\mathrm{CF}}^{-}$states, obtained from extrapolation of data for $N \leq 9$ to $N \rightarrow \infty$.

\section{GENERAL CRITERION FOR SKYRMIONS IN HALF-FILLED SPIN-DEGENERATE SHELL}

Since (i) the finite width $w$ enters the Hamiltonian (1) only through the pseudopotential $V(\mathcal{R})$, (ii) only a few leading parameters $V(0), V(1), V(2), \ldots$ that correspond to a short average $e-e$ distance $\sqrt{\left\langle r^{2}\right\rangle}$ significantly depend on $w$, and (iii) for $w=0$, the opposite behavior of $E_{S}(K)-E_{0}$ for the lowest and excited LL's results precisely from the enhanced value of $V(2)$ for $n \geq 1$, it is enough to study the dependence of the short-range part of $V(\mathcal{R})$ on $w$ to understand the reoccurrence of skyrmions for $n \geq 1$ at $w \sim 2 \lambda$. This dependence is illustrated in Fig. 13(abc). Clearly, increasing $d$ (i.e., $w=5 d)$ suppresses more strongly the pseudopotential parameters at the even values of $\mathcal{R}$ (open circles) corresponding to zero pair spin, specially the highly repulsive ones at $\mathcal{R}=0$ and 2 for $n=1$ and $\mathcal{R}=0,2$, and 4 for $n=2$. While in any LL the strong suppression of $V(0)$ will eventually (at very large $w$ ) destroy skyrmions (all having no pairs with $\mathcal{R}=0$ ), there is a wide range of $w$ in which skyrmions become stable also for $n>0$.

By comparing the values of $w$ and $d$ in Figs. 9 (abc) and 13 (abc), we find the following general conditions for the occurrence of skyrmions in a system of interacting spin- $\frac{1}{2}$ fermions half-filling a spin-degenerate shell: (i) $V(0)$ must be sufficiently large to cause decoupling of the many-body states without the $\mathcal{R}=0$ pairs (skyrmions) from all other many-body states, and (ii) $V(\mathcal{R})$ must decrease with increasing $\mathcal{R} \geq 1$.

Note that the latter condition (ii) is not immediately applicable to shells with broken particle $(\uparrow)-$ hole $(\downarrow)$ symmetry. Examples of such systems include electrons at $\nu=\frac{1}{3}$ (i.e., CF's at $\nu^{*}=1$ ) but also the case when the pair of spin-degenerate LL's has different orbital indices $n$ (which can be realized by making the Zeeman gap 


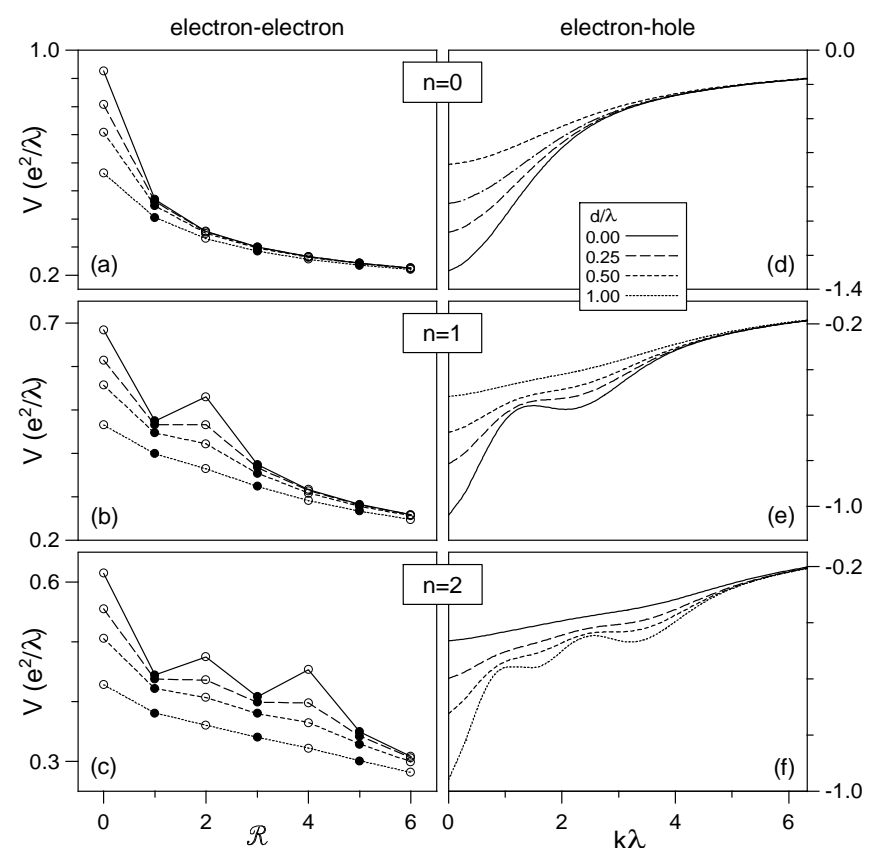

FIG. 13. Pseudopotentials $V$ of the $e-e$ (abc) and $e-h$ (def) interaction in the $n=0$ (ad), 1 (be), and 2 (cf) LL's, calculated using the interaction potential $V_{d}(r)=e^{2} / \sqrt{r^{2}+d^{2}}$ for $d / \lambda=0, \frac{1}{4}, \frac{1}{2}$, and 1 , and describing a quasi-2D layer of width $w=5 d$. $\mathcal{R}$ is relative $e-e$ angular momentum (data shown only for $\mathcal{R} \leq 6$ ), open and closed circles mark singlet and triplet $e-e$ states, respectively, $k$ is the $e-h$ wave vector, and $\lambda$ is the magnetic length.

$E_{Z}$ equal to the cyclotron gap $\hbar \omega_{c}$ in a magnetic material). In these systems, the interaction Hamiltonian (11) is determined by a pair of (different) particle-particle and hole-hole pseudopotentials $V_{\uparrow \uparrow}(\mathcal{R})$ and $V_{\downarrow \downarrow}(\mathcal{R})$, where $\mathcal{R}$ is an odd number (as required for two identical fermions), and a particle-hole continuous dispersion $V_{\uparrow \downarrow}(k)$, where $k$ is the pair wave vector (on a sphere, $k R=L$ ). To rephrase condition (ii) in terms of $V_{\uparrow \downarrow}(k)$, we notice in Fig. 13 that the suppression of the maxima at $\mathcal{R}=2$ (for $n=1$ ) and $\mathcal{R}=2$ and 4 (for $n=2$ ) coincides with the disappearance of the corresponding roton minima in the spin-wave dispersion $V_{\mathrm{eh}}(k)$, at $k \lambda \approx 2.1$ (for $n=1$ ) and $k \lambda \approx 1.5$ and 3.2 (for $n=2$ ).

A continuous evolution of the skyrmion energy spectrum $E_{S}(K)$ from the positive values (as for $n \geq 1$ and small $w$ ) to the negative values (as for $n=0$ or $n \geq 1$ and large $w$ ) can be most easily understood by studying a simple model pseudopotential $U_{x}(\mathcal{R})$ defined as: $U_{x}(0)=\infty, U_{x}(1)=1, U_{x}(2)=x$, and $U_{x}(\mathcal{R})=0$ for $\mathcal{R}>2$. This choice of $U_{x}$ guarantees that skyrmions are its only finite-energy eigenstates, and their energy spectrum $E_{S}(K)$ depends on one free parameter $x$.

The essential information about the skyrmion wave functions iscontained in the fractional grandparentage coefficients 24.25 . The dependence of $\mathcal{G}$ on $\mathcal{R}$ is a pair correlation function defined as a fraction of the $e-e$ pairs with the relative pair angular momentum $\mathcal{R}$. For

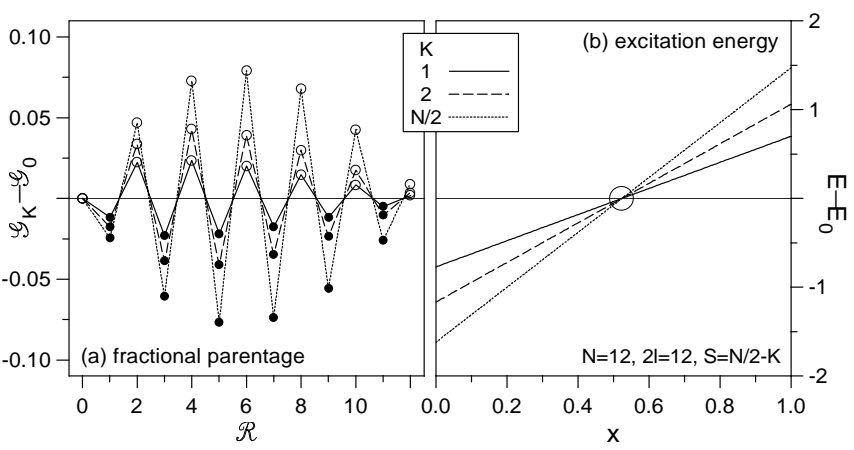

FIG. 14. (a) Pair-correlation functions - fractional grandparentage $\mathcal{G}_{K}$ as a function of relative pair angular momentum $\mathcal{R}$ - for skyrmions with $K=1$ and $\frac{1}{2} N$, calculated for $N=12$ electrons on Haldane sphere. Open and closed circles mark singlet and triplet pair states, respectively. (b) Energy $E$ of the skyrmions with $K=1,2$, and $\frac{1}{2} N$ as a function of parameter $x$ of the model $e-e$ interaction $U_{x}$.

$K=0$, the many-electron system is completely spinpolarized, so that every electron pair has $\operatorname{spin} S=1$, and thus $\mathcal{G}$ vanishes for all even values of $\mathcal{R}$. When $K$ is increased, so does the total fraction of $S=0$ pairs, $\mathcal{G}_{K}(0)+\mathcal{G}_{K}(2)+\ldots$, which happens at the cost of a decreasing number of $S=1$ pairs, $\mathcal{G}_{K}(1)+\mathcal{G}_{K}(3)+\ldots$ The grandparentage coefficients measured from the "reference" value $\mathcal{G}_{0}$ for $K=0$ are plotted in Fig. 14(a) as a function of $\mathcal{R}$ for $K=1$ and $\frac{1}{2} N$. It turns out that $\Delta \mathcal{G}_{K}=\mathcal{G}_{K}-\mathcal{G}_{0}$ is a regular function of $\mathcal{R}$ and, for example, $\Delta \mathcal{G}_{K}(1)=-\alpha \mathcal{G}_{K}(2)$, where $\alpha^{-1}=2-(N-1)^{-1}$. This al using a general expression for the interaction energy,24.25

$$
E=\frac{1}{2} N(N-1) \sum_{\mathcal{R}} \mathcal{G}(\mathcal{R}) V(\mathcal{R})
$$

to write the skyrmion energy for $V=U_{x}$ as

$$
E_{S}(K)-E_{0}=(x-\alpha) \mathcal{G}_{K}(2) .
$$

As shown in Fig. 14(b), $E_{S}(K)-E_{0}$ changes sign simultaneously for all $K$ (the spin-polarized $K=0 \mathrm{QP}$ state becomes unstable toward formation of a skyrmion) when $x=\alpha$, that is when $U_{x}(2)$ drops below $\alpha U_{x}(1)$. Since $\alpha \rightarrow \frac{1}{2}$ for $N \rightarrow \infty$, this means that skyrmions in an infinite (planar) system will have lower energy than a QP state with $G$ when $U_{x}$ becomes superlinear (i.e., superharmonid 24,25) between $\mathcal{R}=1$ and 3 .

\section{CONCLUSION}

We have presented the results of detailed numerical studies of the spin excitations of various ferromagnetic GS's of a 2DEG confined in a quantum well of finite width $w$, in the integral and fractional quantum Hall regime. Similar low-energy spectra in the vicinity of $\nu=1$ and 
$\frac{1}{3}\left(\nu^{*}=1\right.$ in the CF picture) have been found, and both contain the following two types of low-energy excitations: spin waves 66 , and skyrmions and anti-skyrmions 29.50 . The phase diagrams for the occurrence of skyrmions with different numbers of reversed spins $K$ as a function of the well width $w$ and the Zeeman energy $E_{\mathrm{Z}}$ have been determined at $\nu=1,3$, and 5 .

The interactions between the (neutral) spin waves and (charged) skyrmions have also been studied. We found that the spin waves each carrying angular momentum $L=1$ condense into an ordered (with parallel angular momenta), correlated, and noninteracting state. The interaction energy $E_{W}$ of this condensate is a linear function of (continuous) spin polarization $\zeta$ which, in the absence of the Zeeman energy, gives rise to a gapless and continuous density of states. This is in contrast to a discrete spectrum of particle-like skyrmion excitations, whose energy $E_{S}$ is a function of the (integral) reversedspin number $K$. The short-range repulsion between charged skyrmions is predicted to cause Laughlin correlations, that is the tendency to avoid skyrmion pair eigenstates with the smallest relative angular momenta $\mathcal{R}$. This causes effective spatial isolation of skyrmions from one another, and the absence of high-energy skyrmionskyrmion collisions.

The major differences between the $\nu=1$ and $\frac{1}{3}$ spectra are the reduced energy scale and a broken skyrmionanti-skyrmion symmetry in the latter system (broken particle-hole symmetry in the lowest CF LL). A number of phenomena associated with the particular form of spin excitations at $\nu \approx 1$ (rapid depolarization at $\nu=1^{ \pm}$, nonlinear transport through a finite-size droplet, sensitivity of the spin coupling to magnetic particles to $\nu$, etc.) are also expected at $\nu \approx \frac{1}{3}$. The smallest skyrmion and anti-skyrmion states at $\nu=\frac{1}{3}$ are equivalent to composite fermion charged excitons $X_{\mathrm{CF}}^{ \pm}$.

A qualitatively different behavior is observed in the excited LL's. In agreement with earlier theories,51,52 we find that skyrmions and anti-skyrmions are unstable at $\nu=3$ or 5 even at $E_{\mathrm{Z}}=0$, which (in contrast to $\nu=1$ or $\left.\frac{1}{3}\right)$ results in the stability of the ferromagnetic order at all nearby values of $\nu$, and the single-particle character of elementary excitations. In the $e-h$ picture, this means unbinding of charged excitons in the excited LL's. Also in contrast to $\nu=1$ or $\frac{1}{3}$, the $L=1$ spin waves attract one another rather than decouple, which for example results in direct confinement-induced transitions between the consecutive "compact" states of finite $\nu=3$ or 5 quantum Hall droplets, skipping the correlated, depolarized states with intermediate density.

This different behavior in the excited LL's is suppressed when the width $w$ of a quasi-2D layer exceeds about two magnetic lengths. This critical value obtained from a finite-size-calculation seems to agree better with the experiments 53.55 than an earlier estimate 54 The reoccurrence of skyrmions in excited LL's in wider quantum wells is explained by studying the involved particle- particle and particle-hole interaction pseudopotentials and the electron correlations in the skyrmion eigenstates.

A general criterion is found that allows prediction of the presence or absence of skyrmions in a system of interacting spin- $\frac{1}{2}$ fermions in a half-filled spin-degenerate shell. The criterion describes correctly all calculated spin excitation spectra at $\nu=\frac{1}{3}, 1,3$, and 5 , and at arbitrary layer widths $w$, density profile $\varrho(z)$, etc.

\section{ACKNOWLEDGMENT}

The authors acknowledge partial support by the Materials Research Program of Basic Energy Sciences, US Department of Energy. JJQ thanks National Magnetic Field Laboratory, Tallahassee, and University of New South Wales, Sydney, for hospitality. AW acknowledges helpful discussions with P. Hawrylak, L. Jacak, A. H. MacDonald, J. J. Palacios, M. Potemski, and I. Szlufarska, and support from grant 2P03B11118 of the Polish State Committee for Scientific Research (KBN).

${ }^{1}$ The Quantum Hall Effect, edited by R. E. Prange and S. M. Girvin (Springer-Verlag, New York, 1987).

${ }^{2}$ T. Chakraborty and P. Pietiläinen, The Quantum Hall Effects, (Springer-Verlag, New York, 1995).

${ }^{3}$ Perspectives in Quantum Hall Effects, edited by S. Das Sarma and A. Pinczuk, (John Wiley \& Sons, New York, 1997).

${ }^{4}$ R. B. Laughlin, Phys. Rev. Lett. 50, 1395 (1983).

${ }^{5}$ F. D. M. Haldane, in Ref. 1, chap. 8, p. 303.

${ }^{6}$ J. J. Quinn and A. Wójs, J. Phys.: Cond. Mat. 12, R265 (2000).

${ }^{7}$ D. C. Tsui, H. L. Stormer, and A. C. Gossard, Phys. Rev. Lett. 48, 1559 (1982).

${ }^{8}$ S. E. Barrett, G. Dabbagh, L. N. Pfeiffer, K. W. West, and R. Tycko, Phys. Rev. Lett. 74, 5112 (1995).

${ }^{9}$ R. Tycko, S. E. Barrett, G. Dabbagh, L. N. Pfeiffer, and K. W. West, Science 268, 1460 (1995).

${ }^{10}$ D. K. Maude, M. Potemski, J. C. Portal, M. Henini, L. Eaves, G. Hill, and M. A. Pate, Phys. Rev. Lett. 77, 4604 (1996).

${ }^{11}$ E. H. Aifer, B. B. Goldberg, and D. A. Broido, Phys. Rev. Lett. 76, 680 (1996).

${ }^{12}$ M. J. Manfra, E. H. Aifer, B. B. Goldberg, D. A. Broido, L. Pfeiffer, and K. West, Phys. Rev. B 54, R17327 (1996).

13 S. P. Shukla, M. Shayegan, S. R. Parihar, S. A. Lyon, N. R. Cooper, and A. A. Kiselev, Phys. Rev. B 61, 4469 (2000).

${ }^{14}$ P. Khandelwal, A. E. Dementyev, N. N. Kuzma, S. E. Barrett, L. N. Pfeiffer, and K. W. West, Phys. Rev. Lett. 86, 5353 (2001).

15 T. Skyrme, Proc. R. Soc. London A 262, 237 (1961).

${ }^{16}$ R. Ramajaran, Solitons and Instantons, (North-Holland, Amsterdam, 1982). 
17 D. H. Lee and C. L. Kane, Phys. Rev. Lett. 64, 1313 (1990).

${ }^{18}$ F. D. M. Haldane, Phys. Rev. Lett. 51, 605 (1983).

19 J. K. Jain, Phys. Rev. Lett. 63, 199 (1989).

${ }^{20}$ A. Lopez and E. Fradkin, Phys. Rev. B 44, 5246 (1991).

${ }^{21}$ B. I. Halperin, P. A. Lee, and N. Read, Phys. Rev. B 47, 7312 (1993).

${ }^{22}$ P. Sitko, K. S. Yi, and J. J. Quinn, Phys. Rev. B 56, 12417 (1997).

${ }^{23}$ K. von Klitzing, G. Dorda, and M. Pepper, Phys. Rev. Lett. 45, 494 (1980).

${ }^{24}$ A. Wójs and J. J. Quinn, Acta Phys. Pol. A 96, 403 (1999).

${ }^{25}$ A. Wójs and J. J. Quinn, Philos. Mag. B 80, 1405 (2000).

${ }^{26}$ A. Wójs, Phys. Rev. B 63, 125312 (2001).

27 A. Wójs and J. J. Quinn, Phys. Rev. B 61, 2846 (2000).

${ }^{28}$ S. He, F. C. Zhang, X. C. Xie, and S. Das Sarma, Phys. Rev. B 42, R11376 (1990).

29 S. L. Sondhi, A. Karlhede, S. A. Kivelson, and E. H. Rezayi, Phys. Rev. B 47, 16419 (1993).

${ }^{30}$ H. A. Fertig, L. Brey, R. Côté, and A. H. MacDonald, Phys. Rev. B 50, 11018 (1994).

${ }^{31}$ L. Brey, H. A. Fertig, R. Côté, and A. H. MacDonald, Phys. Rev. Lett. 75, 2562 (1995).

${ }^{32}$ K. Moon, H. Mori, K. Yang, S. M. Girvin, A. H. MacDonald, L. Zheng, D. Yoshioka, and S. C. Zhang, Phys. Rev. B 51, 5138 (1995).

${ }^{33}$ A. H. MacDonald, H. A. Fertig, and L. Brey, Phys. Rev. Lett. 76, 2153 (1996).

${ }^{34}$ X. C. Xie and S. He, Phys. Rev. B 53, 1046 (1996).

${ }^{35}$ R. Côté and A. H. MacDonald, Phys. Rev. B 53, 10019 (1996).

${ }^{36}$ R. Côté, A. H. MacDonald, L. Brey, H. A. Fertig, S. M. Girvin, and H. T. C. Stoof, Phys. Rev. Lett. 78, 4825 (1997).

${ }^{37}$ M. Abolfath, J. J. Palacios, H. A. Fertig, S. M. Girvin, and A. H. MacDonald, Phys. Rev. B 56, 6795 (1997).

${ }^{38}$ H. A. Fertig, L. Brey, R. Côté, A. H. MacDonald, A. Karlhede, and S. L. Sondhi, Phys. Rev. B 55, 10671 (1997).

39 J. H. Oaknin, B. Paredes, and C. Tejedor, Phys. Rev. B 58, 13028 (1998).

${ }^{40}$ V. Melik-Alaverdian, N. E. Bonesteel, and G. Ortiz, Phys. Rev. B 60, R8501 (1999).

${ }^{41}$ J. J. Palacios, L. Martin-Moreno, G. Chiappe, E. Louis, and C. Tejedor, Phys. Rev. B 50, 5760 (1994).

42 J. H. Oaknin, L. Martin-Moreno, and C. Tejedor, Phys. Rev. B 54, 16850 (1996).

43 A. Wójs and P. Hawrylak, Phys. Rev. B 56, 13227 (1997).

${ }^{44}$ A. H. MacDonald and E. H. Rezayi, Phys. Rev. B 42, 3224 (1990).

45 A. Wójs and P. Hawrylak, Phys. Rev. B 51, 10880 (1995).

46 J. J. Palacios, D. Yoshioka, and A. H. MacDonald, Phys. Rev. B 54, 2296 (1996).

${ }^{47}$ R. K. Kamilla, X. G. Wu, and J. K. Jain, Solid State Commun. 99, 289 (1996).

${ }^{48}$ D. R. Leadley, R. J. Nicholas, D. K. Maude, A. N. Utjuzh, J. C. Portal, J. J. Harris, and C. T. Foxon, Phys. Rev. Lett. 79, 4246 (1997).

${ }^{49}$ P. Khandelwal, N. N. Kuzma, S. E. Barrett, L. N. Pfeiffer, and K. W. West, Phys. Rev. Lett. 81, 673 (1998).

${ }^{50}$ A. H. MacDonald and J. J. Palacios, Phys. Rev. B 58, R10171 (1998).
51 J. K. Jain and X. G. Wu, Phys. Rev. B 49, 5085 (1994).

${ }^{52}$ X. G. Wu and S. L. Sondhi, Phys. Rev. B 51, 14725 (1995).

53 A. Schmeller, J. P. Eisenstein, L. N. Pfeiffer, and K. W. West, Phys. Rev. Lett. 75, 4290 (1995).

${ }^{54}$ N. R. Cooper, Phys. Rev. B 55, R1934 (1997).

${ }^{55}$ Y. Q. Song, B. M. Goodson, K. Maranowski, and A. C. Gossard, Phys. Rev. Lett. 82, 2768 (1999).

56 A. Wójs, P. Hawrylak, and J. J. Quinn, Phys. Rev. B 60, 11661 (1999).

${ }^{57}$ A. Wójs, I. Szlufarska, K. S. Yi, and J. J. Quinn, Phys. Rev. B 60, R11273 (1999).

58 A. Wójs, J. J. Quinn, and P. Hawrylak, Phys. Rev. B 62, $4630(2000)$

${ }^{59}$ I. Szlufarska, A. Wójs, and J. J. Quinn, Phys. Rev. B 64 (to appear October 15, 2001).

60 T. Chakraborty, P. Pietiläinen, and F. C. Zhang, Phys. Rev. Lett. 57, 130 (1986)

${ }^{61}$ E. H. Rezayi, Phys. Rev. B 36, 5454 (1987); ibid. 43, 5944 (1991).

62 T. T. Wu and C. N. Yang, Nucl. Phys. B 107, 365 (1976).

${ }^{63}$ G. Fano, F. Ortolani, and E. Colombo, Phys. Rev. B 34, 2670 (1986).

${ }^{64}$ J. E. Avron, I. W. Herbst, and B. Simon, Ann. Phys. 114, 431 (1978).

${ }^{65}$ A. Wójs and J. J. Quinn, Physica E 3, 181 (1998).

${ }^{66}$ C. Kallin and B. I. Halperin, Phys. Rev. B 30, 5655 (1984).

${ }^{67}$ A. B. Dzyubenko and Yu. E. Lozovik, Fiz. Tverd. Tela 25, 1519 (1983) [Sov. Phys. Solid State 25, 874 (1983)]. 